\title{
Superconducting nanowire single-photon detection system and demonstration in quantum key distribution
}

\author{
CHEN SiJing ${ }^{1,2}$, LIU DengKuan $^{1,2}$, YOU LiXing ${ }^{1 *}$, WANG YongLiang ${ }^{1}$, QIU LongQing ${ }^{1}$, \\ XIE XiaoMing ${ }^{1}$, JIANG MianHeng ${ }^{1}$, CHEN TengYun ${ }^{3}$, LIU Yang ${ }^{3}$, \\ MIKI Shigehito ${ }^{4} \&$ WANG Zhen ${ }^{4}$ \\ ${ }^{1}$ State Key Laboratory of Functional Materials for Informatics, Shanghai Institute of Microsystem and Information Technology, Chinese Academy \\ of Sciences, Shanghai 200050, China; \\ ${ }^{2}$ University of Chinese Academy of Sciences, Beijing 100049, China; \\ ${ }^{3}$ Hefei National Laboratory for Physical Sciences at Microscale and Department of Modern Physics, University of Science and Technology of \\ China, Hefei 230026, China; \\ ${ }^{4}$ Kobe Advanced Research Center, National Institute of Information and Communications Technology, 588-2, Iwaoka, Nishi-ku, Kobe, Hyogo \\ 651-2492, Japan
}

Received June 3, 2012; accepted September 10, 2012

\begin{abstract}
We developed a superconducting nanowire single-photon detection (SNSPD) system based on Gifford-McMahon cryocooler for quantum communication applications. Environmental factors which may influence the system performance are intensively studied. Those factors include temperature fluctuations, the ambient magnetic field and the background radiation. By optimizing the bias circuit, the stability of SNSPD system to electrical noise and disturbance was effectively enhanced, thus making it more suitable for field application. A 4-channel SNSPD system with quantum efficiency higher than $4 \%$ at the dark count rate of $10 \mathrm{~Hz}$ for $\lambda=1550 \mathrm{~nm}$ is integrated and applied into a quantum key distribution (QKD) experiment. QKD was successfully carried out over $100 \mathrm{~km}$ optical fiber with the final secure key rate of $1.6 \mathrm{kbps}$ and the quantum bit error rate of less than $2 \%$.
\end{abstract}

superconducting nanowire single-photon detection, quantum efficiency, latching, quantum key distribution

Citation: Chen S J, Liu D K, You L X, et al. Superconducting nanowire single-photon detection system and demonstration in quantum key distribution. Chin Sci Bull, 2013, 58: 1145-1149, doi: 10.1007/s11434-013-5698-1

Quantum communication is a new-generation technology based on the theory of quantum mechanics, which may provide absolute security for future communication [1]. It is a hot topic in science and technology of quantum control and now in its critical stage from the theoretical research to practical applications.

Quantum key distribution (QKD) is the kernel of the quantum communication. The implementation of QKD relies on several technologies, including single-photon source, encrypting/decrypting, key storage/transmission and singlephoton detection. QKD in fiber over $100 \mathrm{~km}$ had been reported [2]. However, further improvement is limited by the

*Corresponding author (email: 1xyou@mail.sim.ac.cn) available single-photon detection technique. Traditional semiconducting single-photon detectors (SPD) (such as APD: avalanche photodiode, PMT: photomultiplier tube) cannot fulfill the requirement of long haul quantum communication due to the high dark count rate $\left(R_{\mathrm{DC}}\right)$.

Superconducting nanowire single-photon detector/detection (SNSPD) is a novel single-photon detection technology, which uses a nanowire folded into a meander structure fabricated on superconducting ultrathin films. When a photon hits the nanowire, it may produce a transient voltage pulse, which forms the basis of the detection mechanism of SNSPD. This concept was first proposed by Kadin et al. in 1996 [3] and demonstrated by Gol'tsman et al. in 2001 using SNSPD made of an ultra-thin $\mathrm{NbN}$ film [4]. SNSPD 
surpasses the traditional semiconducting SPDs with lower $R_{\mathrm{DC}}$, higher quantum efficiency $(\mathrm{QE})$, smaller timing jitter $(<100 \mathrm{ps})$ and higher counting rate $(\sim \mathrm{GHz})[5]$. Using the optical cavity and the lensed fiber, system QE reaches $21 \%$ at $1550 \mathrm{~nm}$ wavelength when $R_{\mathrm{DC}}=100 \mathrm{~Hz}$ [6]. SNSPD has been applied into a few QKD experiments due to its outstanding performance. Besides, its advantages were also demonstrated in other fields such as fluorescence spectra of molecules, non-contact VISI chip testing and high energy physics. Another promising superconducting single photon detector is the transition edge sensor (TES) with high QE ( $95 \%$ for an incident wavelength of $1556 \mathrm{~nm}$ [7]) and low $R_{\mathrm{DC}}$. However, its low maximum counting rate (1 $\mathrm{MHz}[8]$ ) and rigorous working temperature $(<100 \mathrm{mK}$ [9]) both make it unsuitable for the applications like quantum communication.

As a device made of low temperature superconducting material, SNSPD has to be operated below the liquid helium temperature. For the application of quantum communication, we carried out the researches on SNSPD system integration based on Gifford-McMahon (GM) cryocooler. In this work, we studied the environmental factors which may influence the system performance, including temperature fluctuations, the ambient magnetic field and the background radiation. Besides, we optimized the bias circuit and improved the system's resistance to the electrical noise and the disturbance. A 4-channel SNSPD system with high performance (QE $\geqslant 4 \% @ R_{\mathrm{DC}}=10 \mathrm{~Hz}$ when $\lambda=1550 \mathrm{~nm}$ ) was successfully integrated and applied into QKD experiments. QKD was successfully carried out with the optical fiber length of 100 $\mathrm{km}$ with the final secure key rate of $1.6 \mathrm{kbps}$ and the quantum bit error rate of less than $2 \%$. This result meets the requirement of real-time voice quantum communication.

\section{SNSPD system}

Based on GM closed recycled cryocooler, we setup a SNSPD system using the devices made from ultra-thin $\mathrm{NbN}$ film. The effective part of the devices is 100-nm-wide meandered nanowires, covering an area of $15 \mu \mathrm{m} \times 15 \mu \mathrm{m}$ with a filling factor of $50 \%$. The typical critical temperature $T_{\mathrm{c}}$ is about $10 \mathrm{~K}$. The GM cryocooler is from Sumitomo Inc. with a nominal cooling power of $0.1 \mathrm{~W} @ 4.2 \mathrm{~K}$. Isolated voltage sources are used to provide a stable bias to SNSPD. The typical bias current $I_{\mathrm{b}}$ is slightly lower than the critical current $I_{\mathrm{c}}$ and supplied through the DC arm of the Bias-Tee which is in series with a $10 \mathrm{k} \Omega$ resistor. When the device receives a photon, a transient electrical pulse is generated and output through the RF arm of the Bias-Tee, and then amplified by a low noise amplifier. The amplified signal is fed into an oscilloscope (Tek DSA71254) or a counter (SR400) for further analysis. The system schematic diagram is shown in Figure 1. In order to characterize QE, a picosecond pulse laser diode (Hamamatsu C10196, the typical pulse duration is $70 \mathrm{ps}$ ) is used as the light source, the input

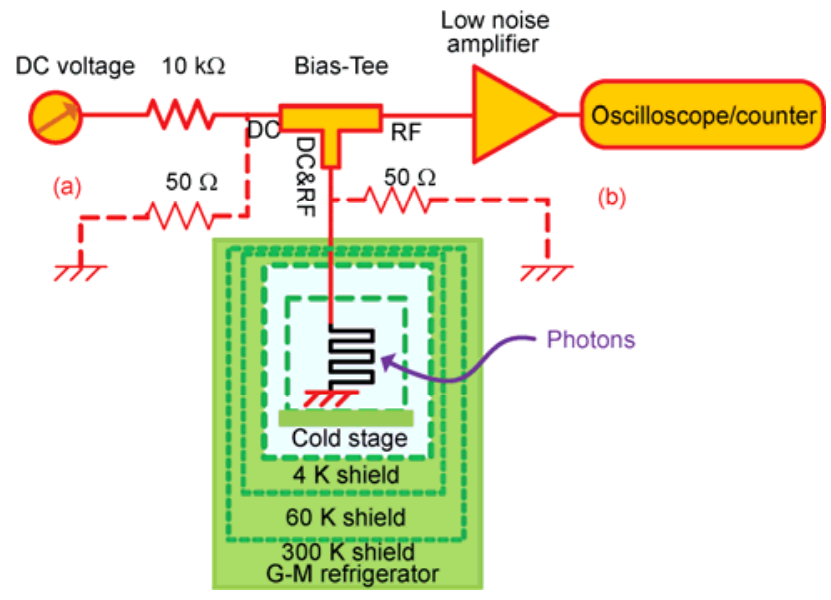

Figure 1 Schematic diagram of the SNSPD system. (a) and (b) indicate 2 different ways of paralleling resistor for optimizing the bias circuit.

light is heavily attenuated by a variable attenuator to achieve a photon flux of 1 photon/pulse, then adjusted by a polarization controller and finally arrives the device through a single-mode fiber. It is noted here that the SNSPD is a polarization sensitive detector [10]. By adjusting the polarization of the incident photons, we may obtain the maximal QE of SNSPD.

\section{Study of SNSPD system performance}

As a superconducting light detection technology with quantum limit sensitivity, the SNSPD system performance not only depends on the performance of the devices, but also closely relates to the working environment. We measured and analyzed the impact of some major environment factors on system's performance, which include temperature fluctuations, the ambient magnetic field and the background radiation. Besides, the electronics are also optimized for improving the system stability.

\subsection{Temperature fluctuation}

As a pulse-compressed refrigerator, the cold stage temperature of the GM cryocooler has an intrinsic periodic fluctuation, which has an amplitude of about $\pm 150 \mathrm{mK}$ and a frequency of $1 \mathrm{~Hz}$. In our previous work, we investigated this original fluctuations and its impact on system's performances in detail. By suppressing the periodic temperature fluctuation (PTF), the system performance can be effectively improved [11].

In our latest experiment the cooling system is improved and the PTF is effectively suppressed to $\pm 10 \mathrm{mK}$. However, a non-periodic temperature fluctuation (nPTF) of the cold stage is observed. Though the origin is unclear, we are able to measure the influence of $\mathrm{nPTF}$ on $R_{\mathrm{DC}}$ by counting the dark counts at a fixed bias current every $2 \mathrm{~s}$. 
Figure 2 shows the results of the temperature fluctuation and $R_{\mathrm{DC}}$ variation over $200 \mathrm{~s}$. The PTF has an amplitude of $10 \mathrm{mK}$. Besides, nPTF drifts over $30 \mathrm{mK}$ in $180 \mathrm{~s}$ and $R_{\mathrm{DC}}$ also varies $2-3$ times. The interesting phenomenon is that the variation of $R_{\mathrm{DC}}$ (in logarithmic coordinate) follows exactly the behavior of nPTF, which clearly proves that dark count is very sensitive to the temperature fluctuation.

The origin of the dark counts is generally thought to be the thermal unbinding of the vortex-antivortex pair (VAP). Indeed, according to the VAP unbinding theory $[12,13], R_{\mathrm{DC}}$ can be expressed as

$$
R_{\mathrm{DC}}(T)=\Gamma_{\mathrm{VAP}} \exp \left(-U_{\mathrm{b}} / k_{\mathrm{B}} T\right) .
$$

Here $\Gamma_{\mathrm{vAP}}$ is the attempt rate, $U_{\mathrm{b}}$ is the minimum binding energy and $k_{\mathrm{B}}$ is the Boltzmann constant. By proper approximation and simplification, eq. (1) can be converted to

$$
\Delta \ln R_{\mathrm{DC}}(T)=\ln R_{\mathrm{DC}}(T+\Delta T)-\ln R_{\mathrm{DC}}(T) \approx \alpha(T) \Delta T .
$$

Here $\alpha(T)$ is a temperature related coefficient. When $\Delta T \ll T, 2.71 \mathrm{~K}<T<2.76 \mathrm{~K}, \alpha(T)$ is nearly a constant and eq. (2) explains very well the behavior in Figure 2.

For the application in QKD, SNSPD system should have the minimum temperature fluctuation to keep stable QE and $R_{\mathrm{DC}}$. Thus how to further suppress PTF and nPTF of SNSPD system is still an unavoidable issue need to be addressed.

\subsection{Ambient magnetic field}

$R_{\mathrm{DC}}$ is one of the key parameters of SNSPD system for QKD. As the origin of dark counts, the thermal unbinding of the VAP is supposed to be related to the ambient magnetic field. However, the direct influence of the ambient magnetic field on SNSPD is not verified. In order to eliminate the possible impact of the ambient magnetic field, the device was often well shielded with $\mu$-metal block, and thus the magnetic field surrounding the device was reduced by

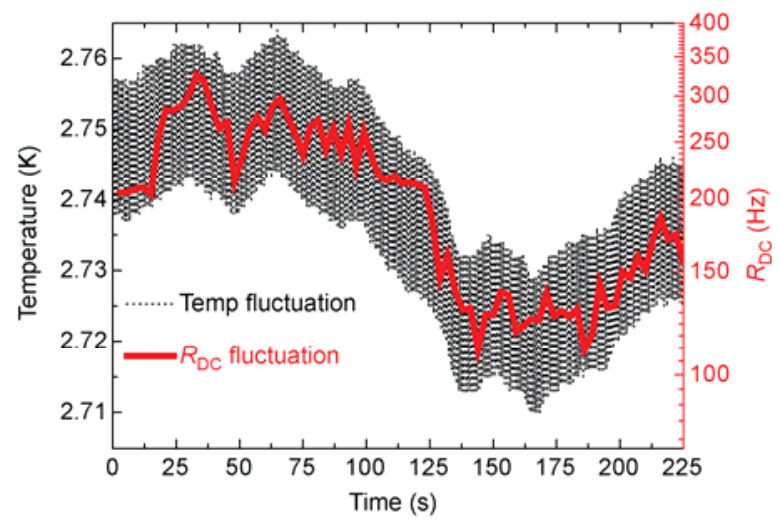

Figure 2 Variation of cold stage's temperature and system $R_{\mathrm{DC}}$ with time. The red solid curve indicates the fluctuation of $R_{\mathrm{DC}}$ and the black dot line indicates the temperature fluctuation. two orders of magnitude [14]. Alternatively, we may use a homemade three-axis Helmholtz coil (shown in Figure 3) to cancel the ambient magnetic field of SNSPD system. This method provides a simple yet direct way to check the difference of $R_{\mathrm{DC}}$ with and without the ambient magnetic field.

First, a tri-axial fluxgate was laid on top of the cryostat which is placed in the center of the three-axis Helmholtz coil. By adjusting the currents in the Helmholtz coil, we may reduce all the three components of the ambient magnetic field $B_{x}, B_{y}$ and $B_{z}$ to the minimum. In our laboratory, the amplitude of the ambient magnetic field was reduced from $40 \mu \mathrm{T}$ to $1 \mu \mathrm{T}$.

We measured $R_{\mathrm{DC}}$ and $\mathrm{QE}$ of the SNSPD system in the two different cases respectively. No evident discrepancy is observed, which proves that ambient magnetic field has no evident influence on the SNSPD system performance.

\subsection{Background radiation}

Blackbody radiation from some parts of the refrigerator with a finite temperature is the main origin of the background radiation. The temperature of the cold stage may rise without good thermal shielding, which results in lower QE and higher $R_{\mathrm{DC}}$. It was proven that the device had better performance with a lower temperature [15]. In SNSPD system, the device is fixed in the oxygen-free copper block which is screwed onto the cold stage. Fibers and coaxial cables have to be connected to the device block through several holes on the $4 \mathrm{~K}$ and $60 \mathrm{~K}$ shield cover. Meanwhile, blackbody radiation from the $300 \mathrm{~K}$ shield cover as well as $60 \mathrm{~K}$ shield cover may also pass through the holes, thus affecting the system cooling efficiency. By improving the system design

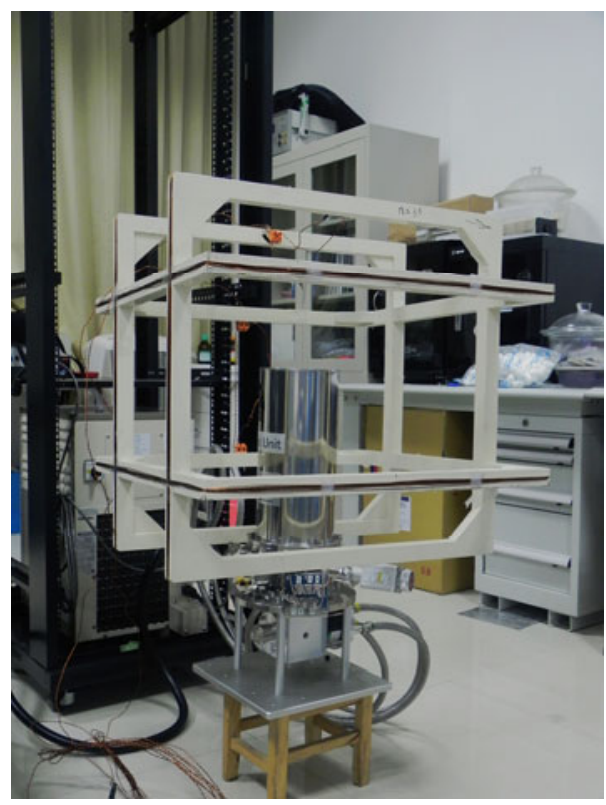

Figure 3 Photograph of the three-axis Helmholtz coil with cryostat in the center. 
with better thermal shielding, the minimum temperature of the cold stage can be decreased from $3.2 \mathrm{~K}$ to $2.5 \mathrm{~K}$ and even lower. As a result, the performance of SNSPD is effectively improved.

\subsection{Electronic noise and/or electric disturbance}

The typical critical current $I_{\mathrm{c}}$ of SNSPD devices is 10-50 $\mu \mathrm{A}$ and the devices often work at a bias current $I_{\mathrm{b}}$ close to $I_{\mathrm{c}}$ $\left(0.95 I_{\mathrm{c}}\right)$. Electronic noise and/or electric disturbance from the power line may cause a current rise $\left|\Delta I_{\mathrm{b}}\right|$ of SNSPD, for example, the switch of a lamp or an air conditioner. When the actual current through SNSPD $I_{b}^{\prime}=I_{\mathrm{b}}+\left|\Delta I_{\mathrm{b}}\right|>I_{\mathrm{c}}$, SNSPD will switch into a resistive state and cannot return to the original superconducting state spontaneously. This process is often referred to as "latching effect" [16]. SNSPD does not response to any incident photons after latching unless we manually reset $I_{\mathrm{b}}$. SNSPD with a lower bias current $I_{\mathrm{b}}$ may decrease the probability of latching caused by the electronic noise and/or the electric disturbance, however, with the sacrifice of QE. Therefore, how to suppress the influence of the electronic noise and/or the electric disturbance on SNSPD system and avoid latching is very important for the practical application of the SNSPD system.

Proper grounding and electronic shielding in laboratory can partially reduce the chance of latching. However, when SNSPD system is imbedded into a QKD network in field test (such as a base station), it is hard to assure good grounding and absolute dc isolation of all the subsystems. Some unexpected disturbance will still cause latching of SNSPD, thus break the unattended long-term operation. For example, switching a fluorescent lamp may produce a surge voltage up to $0.3-1 \mathrm{~V}$ to the bias circuit. Fujiwara et al. [17] added a $50 \Omega$ shunt resistor between the DC\&RF arm of the Bias-Tee and the ground (shown as (b) in Figure 1) to solve the problem. This method can eliminate the latching issue. However, it has two side effects. First, the amplitude of the response pulse is degraded about 30\%. Secondly, the impedance mismatch happens, which results in an extra reflection pulse in the output signal.

We proposed another solution with a resistor paralleling between the DC arm of the Bias-Tee and the ground. The typical resistance is less than $100 \Omega$ (shown in Figure 1). For example, we choose $50 \Omega$ in this work. On the one hand, the resistor reduces the noise from electrical field coupling; on the other hand, it acted as a shunt when electrical disturbance comes, so SNSPD can be stably biased at its superconducting state.

We measured QE, $R_{\mathrm{DC}}$ of SNSPD system and the waveform of the output pulse signal with and without using the new bias circuit. For comparison, we also made the measurements when the resistor was paralleled at (b). No obvious difference was found for $\mathrm{QE}$ and $R_{\mathrm{DC}}$ in these three cases. The waveforms of the output pulse for the three cases are shown in Figure 4. The pulses were almost same for the original bias circuit and the circuit with the resistor paralleled at (a) in Figure 1. However, when the resistor was paralleled at (b) in Figure 1, the pulse's magnitude is suppressed clearly about $30 \%$ from $414 \mathrm{mV}$ to $288 \mathrm{mV}$ and a reflection peak caused by impedance mismatching appeared. The most important improvement is that our solution significantly improves the stability of SNSPD system without any distortions of the output signal, thus producing a longterm running SNSPD system for QKD experiment.

\section{QKD demonstration}

Based on the studies above, the SNSPD system performance is further improved and a 4-channel SNSPD system is integrated with the minimum working temperature lower than $2.5 \mathrm{~K}$. The PTF is $\pm 5 \mathrm{mK}$ and the nPTF is less than $5 \mathrm{mK}$. For $\lambda=1550 \mathrm{~nm}$, all channels satisfy $\mathrm{QE} \geqslant 4 \%$ with $R_{\mathrm{DC}} \leqslant 10$ $\mathrm{Hz}$. The performance QE vs. $R_{\mathrm{DC}}$ of each channel is shown in Figure 5. The performance difference of four channels is owing to different detectors in the four channels.

We applied this 4-channel SNSPD system into the laboratory QKD platform with the fiber length of $100 \mathrm{~km}$. The QKD platform adopted a Decoy-state method and the platform description is referred to [2]. The QKD experiment had worked continuously for 16 hours with SNSPD system installed. The final secure key rate was $1.6 \mathrm{kbps}$ with the quantum bit error less than $2 \%$.

The generated final secure key rate can be estimated by

$$
R=f \times \mu \times \alpha \times \mathrm{QE} \times \beta \times \gamma,
$$

where $R$ indicates the final secure key rate; $f$ represents the working frequency of the light pulse; $\mu$ is the mean photon number per pulse; $\alpha$ indicates the attenuation in the fiber; $\beta$ is the reservation ratio for basis vector comparison; $\gamma$ indicates

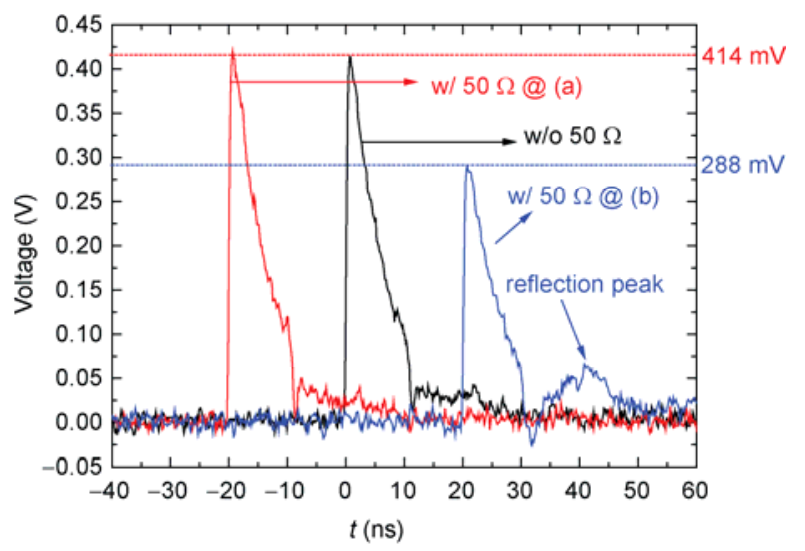

Figure 4 Waveforms of the response pulse in three cases. The red line indicates the circuit with $50 \Omega$ resistor paralleled at (a); the black line indicates the pulse with the original circuit; the blue line indicates the result with $50 \Omega$ resistor paralleled at (b). 


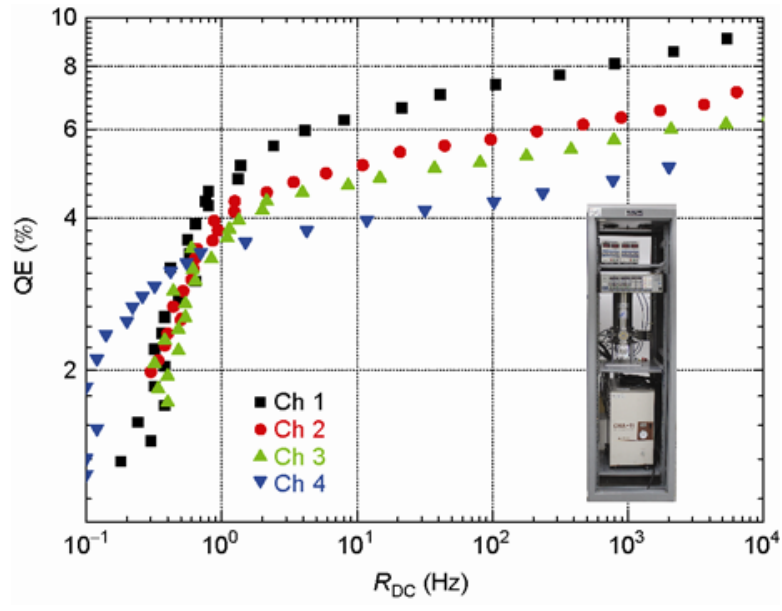

Figure $5 \mathrm{QE}$ as a function of $R_{\mathrm{DC}}$ for all the four channels of the SNSPD system. The inset is the photograph of the 4-channel integrated SNSPD system.

key rate generation factor.

In this experiment, $f=500 \mathrm{MHz}, \mu=0.35, \alpha=0.034$ (the losses of $100 \mathrm{~km}$ length fiber is $26 \mathrm{~dB}$ ), QE=4\%, $\beta=0.5$ and $\gamma$ is $10 \%-30 \%$ typically for this experiment. As a result, the calculated key rate is $1.12-3.36$ kbps. The experimental result is consistent with the calculation. As we know, the minimum bit rate for real-time voice communication is 1.2 $\mathrm{kbps}$. Therefore, our SNSPD system fulfills the requirement of real-time voice quantum communication over $100 \mathrm{~km}$ fiber.

\section{Conclusions}

We intensively studied a series of environmental factors which may influence the SNSPD system performance. Those factors include temperature fluctuations, ambient magnetic and background radiation. With optimizing the bias circuit, SNSPD system's stability is greatly enhanced. These studies are of great significance for the practical application of the SNSPD system. We realized a 4-channel SNSPD system with high performance $\left(\mathrm{QE} \geqslant 4 \%\right.$ @ $R_{\mathrm{DC}}=10$ $\mathrm{Hz}$ when $\lambda=1550 \mathrm{~nm}$ ) and applied it into QKD demonstration over $100 \mathrm{~km}$ fiber successfully. The final secure key rate was $1.6 \mathrm{kbps}$ with the quantum bit error rate less than $2 \%$. The SNSPD system meets the needs of the real-time voice quantum communication over $100 \mathrm{~km}$ fiber.
This work was supported by the National Natural Science Foundation of China (91121022), Strategic Priority Research Program (B) of the Chinese Academy of Sciences (XDB04010200 and XDB04020100), National Basic Research Program of China (2011CBA00202) and National High-Tech Research and Development Program of China (2011AA010802).

1 Gisin N, Ribordy G, Tittel W, et al. Quantum cryptography. Rev Mod Phys, 2002, 74: 145-195

2 Liu Y, Chen T Y, Wang J, et al. Decoy-state quantum key distribution with polarized photons over $200 \mathrm{~km}$. Opt Express, 2010, 18: 8587-8594

3 Kadin A M, Johnson M W. Nonequilibrium photon-induced hotspot: A new mechanism for photodetection in ultrathin metallic films. Appl Phys Lett, 1996, 69: 3938-3940

4 Gol'tsman G N, Okunev O, Chulkova G, et al. Picosecond superconducting single-photon optical detector. Appl Phys Lett, 2001, 79: 705-708

5 Korneev V, Kouminov P, Matvienko V, et al. Sensitivity and gigahertz counting performance of $\mathrm{NbN}$ superconducting single-photon detectors. Appl Phys Lett, 2004, 84: 5338-5341

6 Miki S, Yamashita T, Fujiwara M, et al. Multichannel SNSPD system with high detection efficiency at telecommunication wavelength. Opt Lett, 2010, 35: 2133-2135

7 Lita A E, Miller A J, Nam S W, et al. Counting near-infrared single-photons with 95\% efficiency. Opt Express, 2008, 16: 3032-3040

8 Fukuda D, Fujii G, Numata T, et al. Photon number resolving detection with high speed and high quantum efficiency. Metrologia, 2009, 46: S288-S292

9 Fukuda D, Fujii G, Numata T, et al. High-efficiency photon-number-resolving detectors based on Hafnium transition-edge sensors. In: The Tenth International Conference on QCMC, Brisbane, Queensland, Australia, 2010

10 Anant V, Kerman A J, Dauler E A, et al. Optical properties of superconducting nanowire single-photon detectors. Opt Express, 2008, 16: 10750-10761

11 Shen X F, You L X, Chen S J, et al. Performance of superconducting nanaowire single photon detection system with different temperature variation. Cryogenics, 2010, 50: 708-710

12 Engel A, Semenov, Hubers $\mathrm{H}$ W, et al. Fluctuation effects in superconducting nanostrips. Physica C, 2006, 444: 12-18

13 Yamashita T, Miki S, Makise K, et al. Origin of intrinsic dark count in superconducting nanowire single photon detectors. Appl Phys Lett, 2011, 99: 161107

14 Kitaygorsky J, Komissarov I, Jukna A, et al. Dark counts in nanostructured $\mathrm{NbN}$ superconducting single-photon detectors and bridges. IEEE Trans Appl Supercond, 2007, 17: 275-278

15 Yamashita T, Miki S, Qiu W, et al. Temperature dependent performances of superconducting nanowire single-photon detectors in an ultralow-temperature region. Appl Phys Express, 2010, 3: 102502102504

16 Kerman A J, Yang J K W, Molnar R J, et al. Electrothermal feedback in superconducting nanowire single-photon detectors. Phys Rev B, 2009, 79: 100509-100512

17 Fujiwara M, Tanaka A, Takahashi S, et al. Afterpulse-like phenomenon of superconducting single photon detector in high speed quantum key distribution system. Opt Express, 2011, 19: 19562-19571

Open Access This article is distributed under the terms of the Creative Commons Attribution License which permits any use, distribution, and reproduction in any medium, provided the original author(s) and source are credited. 